\title{
32 Abnormalities of middle ear function
}

A Loss of the transformer mechanism alone produces a hearing loss of about $50 \mathrm{~dB}$.

B A round window baffle effect in modified radical mastoidectomy may allow a hearing threshold within $25 \mathrm{~dB}$ of normal.

C A columellar effect is produced by conservation of only the ossicular chain lever ratio of the transformer mechanism.

D No sound is perceived by air conduction if there is total loss of the middle ear mechanism.

E A blast injury may increase middle ear compliance.

\section{Middle ear muscles}

A Reflex contraction to sound stimulus is ipsilateral.

B Contractions may be audible.

C The stapedius and tensor tympani produce opposite effects on the drumhead.

D Contraction attenuates the middle and high frequencies.

E Contraction allows protection against acoustic trauma due to explosions.

\section{Hearing by bone conduction}
A The skull vibrates.
B The mandible may have a role.
C Is used as a measure of cochlear function.
D The compression theory applies to hearing sounds of higher frequencies.
E Below $800 \mathrm{~Hz}$ the skull vibrates as a whole. 